\title{
SLGC: A New Cluster Routing Algorithm IN WIRELESS SENSOR NETWORK FOR DECREASE ENERGY CONSUMPTION
}

\author{
Arash Ghorbannia Delavar, Somayeh Shamsi, Nafiseh Mirkazemi, Javad Artin \\ Department of Computer, Payame Noor Universtiy, Tehran, IRAN \\ a_ghorbannia@pnu.ac.ir \\ somayeh_sh64@yahoo.com \\ n.mirkazemi@gmail.com \\ javad.artin@gmail.com
}

\begin{abstract}
Decrease energy consumption and maximizing network lifetime are important parameters in designing and protocols for wireless sensor network (WSN).Clustering is one of the efficient methods in energy consumption by Cluster-Head in WSN. Besides, CH can process and aggregate data sent by cluster members, thus reducing network traffic for sending data to sink. In this paper presents a new cluster routing algorithm by dividing network into grids. In each grid computes the center-gravity and threshold of energy for selecting the node that has the best condition base on these parameters in grid for selecting Cluster-Head in current round, also SLGC selecting Cluster-Heads for next rounds thereby this CHs reduce the volume of controlling messages for next rounds and inform nodes for sending data into $\mathrm{CH}$ of respective round. This algorithm prolong network lifetime and decrease energy consumption by selecting $\mathrm{CH}$ in grid and sending data of grid to sink by this $\mathrm{CH}$. Result of simulation shows that SLGC algorithm in comparison with the previous clustering algorithm has maximizing network lifetime and decrease energy consumption in network.
\end{abstract}

\section{KEYWORDS}

Clustering, Grid, Threshold Energy, Distance, Center-gravity \& Cluster-Head.

\section{INTRODUCTION}

Recent promotions in Electronic and wireless communications industries, has created the ability of designing low consumption, small size, low price and multi applications sensors. These small sensors have the ability of some acts such as receiving, process and sending the environmental data (based on the sensor type), and have created an idea for creation and development of networks that are named WSN [1]. The sensor networks architecture's performance is so that the sensors distribute randomly and uniformly in a region and detect control and process the events, and then they send the information to the Sink Station [2]. Whereas the sensors have high performance, they have some restrictions while developing in high scale. The sensors restrictions can be classified in some categories such as short radio broadcast, band width limitation, low energy power and no battery replacement conditions in most cases. When these restrictions should be managed in an environment including many nodes, so many challenges may be happened [3].Every sensor nodes usually is equipped by a wireless transceiver, a microcontroller

DOI : $10.5121 /$ ijcsea.2012.2304 
International Journal of Computer Science, Engineering and Applications (IJCSEA) Vol.2, No.3, June 2012

and power supplier (a battery). Sensors are generally equipped with data processing and communication capabilities [15]. In wireless sensor networks (WSN), energy efficiency is an important issue $[18,19]$, and what to be done to this issue directly affects the network lifetime of WSN [20]. Hierarchical routing is an efficient way to lower energy consumption within a cluster. Due to the characteristics of wireless channels, multi-hop communications between a data source and a data sink are usually more energy efficient than direct transmission. However, because the cluster heads $(\mathrm{CHs})$ closer to the data sink are burdened with heavy relay traffic, they drain much faster than other CHs. [21]

Some features of sensor network make it different from other wireless and traditional networks. These features are:

- Hardware bottleneck including size's restrictions, power supplier, process power and capacity

- Memory

- So many nodes

- High density of node's distributions in operation region

- Failure talent of the nodes

- Dynamic and periodic topological changes

- Using the broadcast communication instead of peer to peer method

- Data oriented network (The nodes don't have any identification code)

Protocols and sensor networks algorithm should have ability of self-organizing. Thus, routing protocols design should utilize maximum node's energy and increase network lifetime. Also, WSN routing networks should be simple and have few calculation's complexity, optimum energy consumption, few delay in data transferring from nodes to the Sink. In this regard, so many algorithms and protocols have been designed. One of these algorithms is hierarchical method. Aid of hierarchical methods is network clustering to several grids that each grid is managed by a Cluster-Head. By this method, many data aggregation methods could be done in each cluster. Clustering methods have some advantages. The first one is that they divide network to some different grids and each grid is managed by a Cluster-Head. The second one is to decreasing data redundancy because of data aggregation by the Cluster-Head. Sensor networks organization in clustered architecture lead to many clustering algorithm at recent years [4].In fact, Cluster-Heads can process, filter and aggregate data send by cluster members, then reducing network alleviating the bandwidth. Clustering saves energy power. [5, 6] The deployment of wireless sensor networks in many application areas requires self-organization of the network nodes into clusters. Clustering is a network management technique, since it creates a hierarchical structure over a flat network. [17]

In this paper we have presents a clustering algorithm that has clustering network into grids and sending data by the $\mathrm{CH}$ in each cluster thus increase network lifetime and decrease energy consumption. Also in SLGC the data of each cluster is send to BS via a single method. In section 3 we present Energy model of network, in section 4 describe SLGC algorithm and in section 5 show the results of simulation.

\section{RELATED WORKS}

Clustering Algorithm uses for gridding nodes and increasing of network lifetime. In each grid select one of the member nodes as a Cluster-Head and coordinator of actions in each cluster for example aggregation of data [7]. 
The clustering methods can be categorized into static and dynamic in sensor networks. The static methods aim at minimizing the total energy spent during the formation of the clusters for a set of given network parameters, such as the number of nodes in the network [8] The dynamic clustering methods deal with the same energy efficiency problem as the static ones but target for a set of changing network parameters, such as the number of active nodes or the available energy levels in a network [9]. One of the most famous clustering algorithms is LEACH [10]. LEACH is a distributed, proactive, dynamic algorithm that forms clusters of sensors based on the received signal strength and uses local $\mathrm{CHs}$ as routers to the sink [16].In LEACH (low-energy adaptive clustering hierarchy) the position of a $\mathrm{CH}$ was rotated among the nodes within a cluster depending on their remaining energy levels. It was assumed that the number of active nodes in the network and the optimal number of clusters to be formed were parameters that could be programmed into the nodes a priori. The LEACH algorithm is divided into rounds and each round separated into two phases, the set-up phase and the steady-state phase. Set-up phase including select the Cluster-Head for current round. Selecting Cluster-Head is based on the threshold T (n) given by:

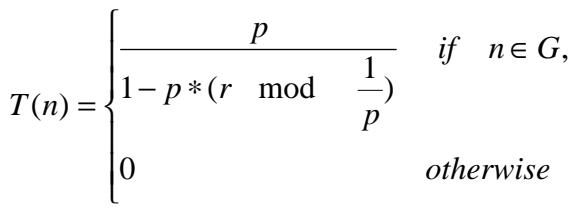

Where $\mathrm{p}$ is the predetermined percentage of cluster heads (e.g., $\mathrm{p}=0.05$ ), $\mathrm{r}$ is the current round, and $\mathrm{G}$ is the set of nodes that have not been cluster heads in the last $1 / \mathrm{p}$ rounds. Cluster head broadcasts an advertisement message to the other nodes. Depending on the signal strength of the advertisement messages, each node selects the cluster head it will belong to. The cluster head creates a Time Division Multiple Access (TDMA) scheme and assigns each node a time slot. In the steady-state phase, the cluster heads collect data from sensor nodes, aggregate the data and send it to the base station. Since the decision to change the $\mathrm{CH}$ is probabilistic, there is a good chance that a node with much low energy gets selected as a $\mathrm{CH}$. When this node dies, the whole cell becomes dysfunctional. Also, the $\mathrm{CH}$ is assumed to have a long communication range so that the data can reach the base-station from the $\mathrm{CH}$ directly. This is not always a realistic assumption since the $\mathrm{CHs}$ are regular sensors and the base-station is often not directly reachable to all nodes due to signal propagation problems. [11]

RCSDN is created based on rounds, in which each round contains two phases, setup and steadystate. In Steady-state Phase the nodes send the data they've received from the environment to the corresponding $\mathrm{CH}$ and after gathering data in the cluster head, data will be sent to the BS. The Set-up Phase in RCSDN starts by sending a start message via BS with a specified range to the environment. After a node receives the start message from the BS, it provides a relative estimate of its distance from the BS through the intensity of the received signal. Then it broadcasts a message for its neighboring nodes including ID, the distance to BS, and the level of remaining energy. Nodes bound in the radio range of this message, receive it and set this node as a neighbor node, and register its ID and energy level in their memory; again they proceed to estimate their distance with the neighboring node by calculating the intensity of the received signal and finally calculate their distance from the BS. This is the done by all nodes in the network and all nodes will acquire the ID, level of energy and distance of all neighboring nodes. In RCSDN we use a local threshold detector (Td) so that only nodes having selection conditions participate in competition for selecting $\mathrm{CH}$. This threshold detector is locally calculated in each node to prevent the lack-of candidates' problem in some areas because of the central selection. [12]. In VIP [13] as sensor networks are deployed over various terrains, the complexity of their topology continues to grow. Voids in networks often cause existing geographic routing algorithms to fail. One 
algorithm for solving this problem is "virtual position", which is the first level of middle Position of all direct neighbors of a node. The middle position is:

$$
\left(\mathrm{x}_{\mathrm{A}}^{\prime}, \mathrm{y}_{\mathrm{A}}^{\prime}\right)=\left(\frac{1}{n} \sum_{i=1}^{n} \mathrm{X}_{\mathrm{A}, \mathrm{i}}, \frac{1}{n} \sum_{i=1}^{n} \mathrm{Y}_{\mathrm{A}, \mathrm{i}}\right)
$$

In this paper we produce the algorithm that use clustering and middle Position of nodes and threshold Energy for finding Cluster-Head $(\mathrm{CH})$ in each grid.

\section{Network Model ANd ENERgy Model}

\subsection{Network Model}

The network model of this paper following this case:

- The BS and all nodes are stationary after deployment of Network.

- The initial energy of nodes is different and randomly.

- Nodes deployment in 2D space.

- Each node has information of coordinate and energy of itself and position of $\mathrm{CH}$.

\subsection{Energy Model}

Sensors consume energy when they sense, receive and transmit data [14].Our energy model for the sensor network is based on the first order radio model assured [10]. In this model, the transmitter has power control abilities to dissipate minimal energy to send data to the receiver. In order to achieve an acceptable signal-to-noise ratio (SNR), the energy consumption of the transmitter is given by:

$$
\begin{cases}E_{T x}(n, d)=n\left(E_{\text {elec }}+\varepsilon_{f s} d^{2}\right) & d<d_{0} \\ E_{T x}(n, d)=n\left(E_{\text {elec }}+\varepsilon_{m p} d^{4}\right) & d \geq d_{0}\end{cases}
$$

Where, $\mathrm{n}$ is the number bit of the message and $\mathrm{d}$ is the distance. $\mathrm{E}_{\text {elec }}$ is the energy dissipated per bit to run the transmitter or the receiver circuit, and ${ }^{\varepsilon_{s s}}, \varepsilon_{m p}$ is the energy dissipated per bit to run the transmit amplifier depending on the distance between the transmitter and receiver. If the distance is less than a threshold $\mathrm{d} 0$, the free space (FS) model is used; otherwise, the multi path (MP) model is used. The energy consumption of the receiver is given by:

$$
E_{R x}(n)=n\left(E_{\text {elec }}\right)
$$

\section{DESCRIPTION OF SLGC ALGORITHM}

SLGC algorithm such as a RCSDN algorithm [12] is created based on rounds and divided into two phases, setup and steady-state. In the Set-up Phase, the $\mathrm{CHs}$ are determined and the cluster structures are formed. In Steady-state Phase the nodes send the data they've received from the neighbor nodes to the $\mathrm{CH}$ and after gathering data in the $\mathrm{CH}$, data will be sent to the BS. In SLGC algorithm Energy and center-gravity are important parameters. 
International Journal of Computer Science, Engineering and Applications (IJCSEA) Vol.2, No.3, June 2012

\subsection{Setup Phase}

In Setup Phase we specified several $\mathrm{CHs}$ for use to different rounds for increasing network lifetime. The Sink (BS) sent initial message for start network into all nodes and all nodes send the information that are useful for running network into Sink. The Sink (BS) after gathering initial data from nodes divides network into some grids based on network size and number of needed clusters. (Figure 1)

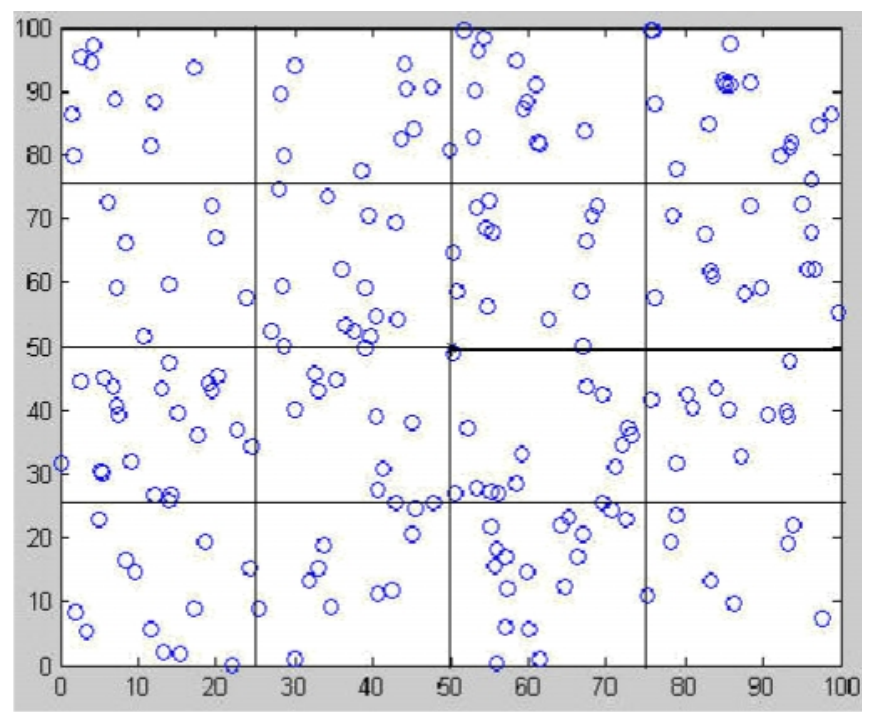

Figure1. Gridding network

The Cluster-Heads should be specified after formation of the clusters. For finding CHs, at first the threshold energy of nodes in each grid should be measured and all nodes that have less energy should be ignored from the candidates list. ( $\alpha$ is a rate that will be specified by the simulator)threshold energy will be calculated by following equation:

$$
\mathrm{E}_{\theta}=\alpha^{*}\left(\frac{1}{n} \sum_{i=1}^{n} \mathrm{E}_{\mathrm{i}}\right)
$$

Following code should be performed for finding the initial candidate nodes set.

\section{Pseudo code of selecting candidate node of energy}

1. If $\mathrm{E}_{\mathrm{i}} \geq \mathrm{E}_{\theta}$ then

2. Insert node $\mathrm{i}$ into $\mathrm{T}$ set $/ / \mathrm{T}$ is a set of nodes that select for candidate nodes in cluster

3. $\quad \mathrm{i}($ type $)=$ candid

$\mathrm{T}$ is a set of nodes that their energy level is higher than the threshold and select for candidate nodes in grid. After adding node into $\mathrm{T}$ set the flag type of node change to a Candid type. Based on SLGC algorithm selecting CHs are based on energy and distance of the node to center-gravity of grid, therefore after filtering nodes base on energy in grid, should be measuring node's centergravity that related to the node's location in each clusters for finding $\mathrm{CHs}$ for several rounds, based on the following equation: 
International Journal of Computer Science, Engineering and Applications (IJCSEA) Vol.2, No.3, June 2012

$$
\left(\mathrm{x}_{\mathrm{A}}^{\prime}, \mathrm{y}_{\mathrm{A}}^{\prime}\right)=\left(\frac{1}{n} \sum_{i=1}^{n} \mathrm{X}_{\mathrm{i}}, \frac{1}{n} \sum_{i=1}^{n} \mathrm{Y}_{\mathrm{i}}\right)
$$

Next step is to specifying the CHs for different rounds in each cluster. At this step, sort $\mathrm{T}$ set ascending base on nodes that have the minimum distance to Gravity, and then nodes that have the less distance to center-gravity are selected as the $\mathrm{CH}$ for different rounds based on the specific rounds number from the sorted list. After find $\mathrm{CHs}$ for different rounds, save information to grid's table. Following code should be performed for finding the final CHs set for each grids:

\section{Algorithm 1 Cluster formation and find CHs in one grid}

1. If $(\bmod ($ round_num, $\operatorname{rmax})=0 \quad / / \mathrm{rmax}$ : the number of rounds for selecting CHs for that

2. $\quad \mathrm{E}_{\theta}=\alpha^{*}\left(\frac{1}{n} \sum_{i=1}^{n} \mathrm{E}_{\mathrm{i}}\right) \quad$ //for all nodes in grid

3. $\mathrm{CG}=\left(\frac{1}{n} \sum_{i=1}^{n} \mathrm{X}_{\mathrm{i}}, \frac{1}{n} \sum_{i=1}^{n} \mathrm{Y}_{\mathrm{i}}\right) \quad$ //for all nodes in grid

4. For $\mathrm{i}=1$ to $\mathrm{n}$

5. If $\mathrm{E}_{\mathrm{i}} \geq \mathrm{E}_{\theta}$ then

6. Insert node $\mathrm{i}$ into $\mathrm{T}$ set $\quad / / \mathrm{T}$ : set of nodes that select for candidate nodes in grid

7. $\mathrm{i}($ type $)=$ candide

8. $\quad$ Distance $(\mathrm{i})=\operatorname{sqrt}\left(\left(\mathrm{X}_{\mathrm{i}}-\mathrm{X}_{\mathrm{CG}}\right)^{2}+\left(\mathrm{Y}_{\mathrm{i}}-\mathrm{Y}_{\mathrm{CG}}\right)^{2}\right)$

9. End

10. Sort $\mathrm{T}$ set ascending base on minimum distance to gravity

11. $\mathrm{CH}=\min (\mathrm{T})$

$/ /$ select $\mathrm{CH}$ for current round

12. For next rounds base on rmax, select CHs base on minimum distance on sorted list Follow chart shows the stages of selecting CHs for different rounds. (Figure 2) 


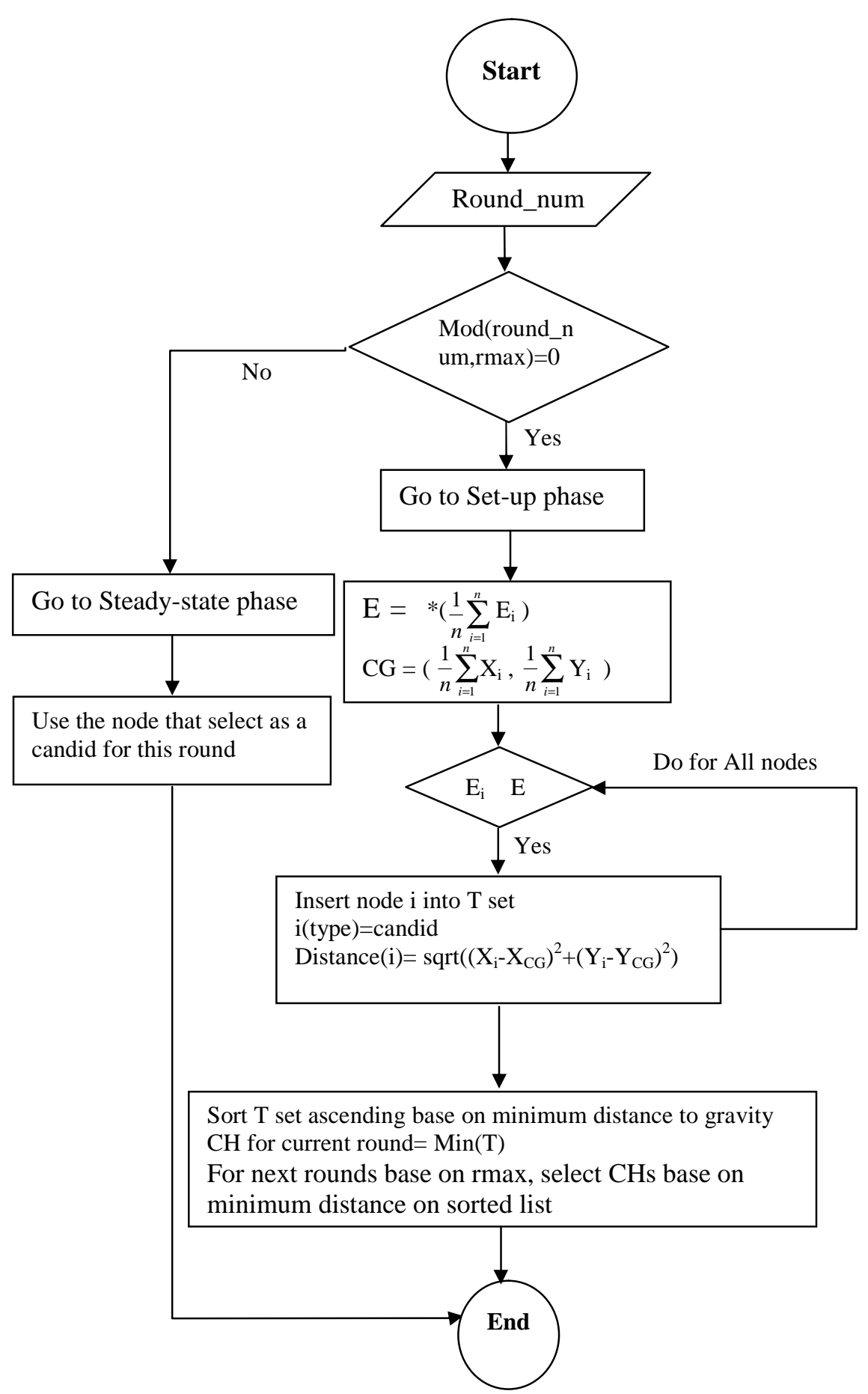

Figure 2. Chart of selecting $\mathrm{CHs}$ 
International Journal of Computer Science, Engineering and Applications (IJCSEA) Vol.2, No.3, June 2012

\subsection{Steady-state Phase}

After sending and aggregating data in current round, the energy of $\mathrm{CH}$ and nodes are decreased, hence the threshold energy base on distance of nodes in grid into $\mathrm{CH}$ is decreased. Base on SLGC algorithm after execute of each round for selecting $\mathrm{CH}$ for next round after calculating new threshold energy if the energy of current round is higher than threshold, current $\mathrm{CH}$ remain $\mathrm{CH}$ for next round and all $\mathrm{CH}$ selected for next round shift one level down. This $\mathrm{CH}$ remain such as $\mathrm{CH}$ because has best situation among all candidate node in grid base on distance to gravity. This idea in SLGC algorithm reduces the volume of controlling messages for next rounds and increase network life time. Following code should be performed for set $\mathrm{CH}$ for next rounds:

\section{Pseudo code of set $\mathrm{CH}$ for next rounds}

//Calculate new threshold energy of grid

1. $\mathrm{E}_{\theta(\text { new })}=\mathrm{E}_{\theta(\mathrm{old})}-\left(\right.$ efs $\left.*(\text { sum of distance node to } \mathrm{CH})^{2}\right) \quad$ //efs: Transmit Amplifier types

2. If $\mathrm{E}_{\mathrm{CH} \text { (previous round) }} \geq \mathrm{E}_{\theta \text { (new) }}$

3. $\mathrm{CH}=\mathrm{CH}($ previous round)

4. Else

5. $\mathrm{CH}=\mathrm{CH}($ next $\mathrm{CH}$ from sorted $\mathrm{T}$ set $)$

All nodes should send their data to the Cluster-Head for aggregating and sending data to the BS, after specifying the candidate node.

\section{Simulation}

We simulated this algorithm; the parameters that use for simulation of this algorithm are as following table. The position of all nodes in coordinate is randomly.

Table 1. Simulation Parameters

\begin{tabular}{|l|l|}
\hline Parameter & Value \\
\hline Network size & $100 * 100 \mathrm{~m}$ \\
\hline Number of nodes & 200 \\
\hline Base station location & $50,50 \mathrm{~m}$ \\
\hline Initial energy for node & rand $[0.5,1] \mathrm{J}$ \\
\hline Eelec & $50 \mathrm{~nJ} / \mathrm{bit}$ \\
\hline$\varepsilon_{f s}$ & $10 \mathrm{pj} / \mathrm{bit} / \mathrm{m} 2$ \\
\hline$\varepsilon_{m p}$ & $0.0013 \mathrm{pj} / \mathrm{bit} / \mathrm{m} 4$ \\
\hline Data aggregation energy & $5 \mathrm{nj} / \mathrm{bit} /$ signal \\
\hline $\mathrm{d}_{0}$ & $87 \mathrm{~m}$ \\
\hline
\end{tabular}

SLGC algorithm have compare with the LEACH [10] method. We use the value of $\alpha=1.05$ to compare our algorithm to LEACH. 
International Journal of Computer Science, Engineering and Applications (IJCSEA) Vol.2, No.3, June 2012

\subsection{The Effect of $\alpha$ Coefficient in Algorithm Efficiency}

We have investigated the effect of $\alpha$ coefficient in algorithm efficiency, $\alpha$ is a coefficient for specified threshold energy for finding candidate node in grids. As figure 3 shows, increasing $\alpha$ don't have good efficiency for network lifetime. We can see that for $\alpha=1.05$ an optimal value of network lifetime can be obtained.

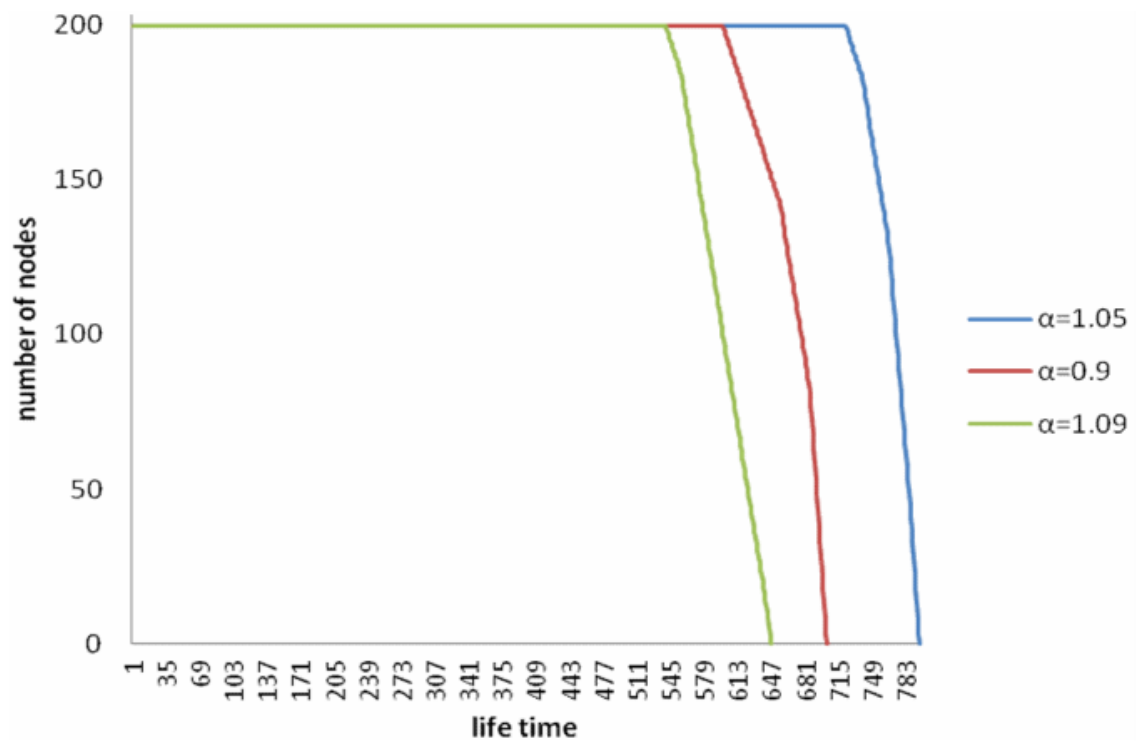

Figure 3. Effect of $\alpha$ coefficient

\subsection{The Numbers of Create Grids}

As show figure 4 and 5, finding CHs in LEACH and SLGC are different. In SLGC for reducing control messages we select $\mathrm{CHs}$ for some rounds base on best position in grid for decreasing energy consumption.

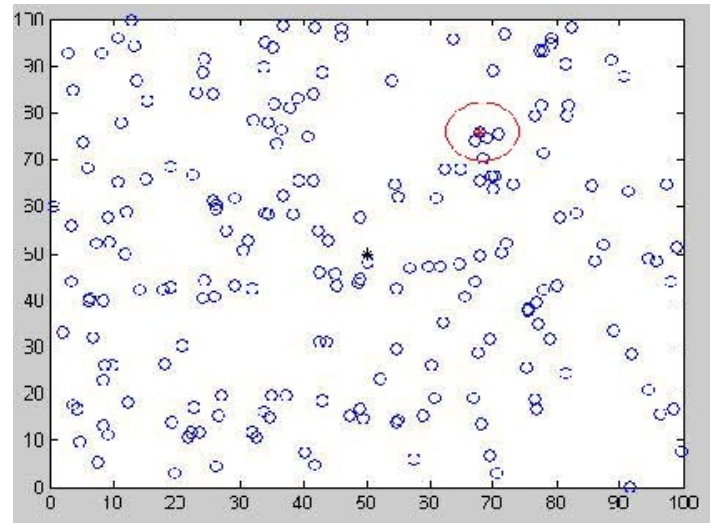

Figure 4. Gridding and find $\mathrm{CH}$ in $\mathrm{LEACH}$ 


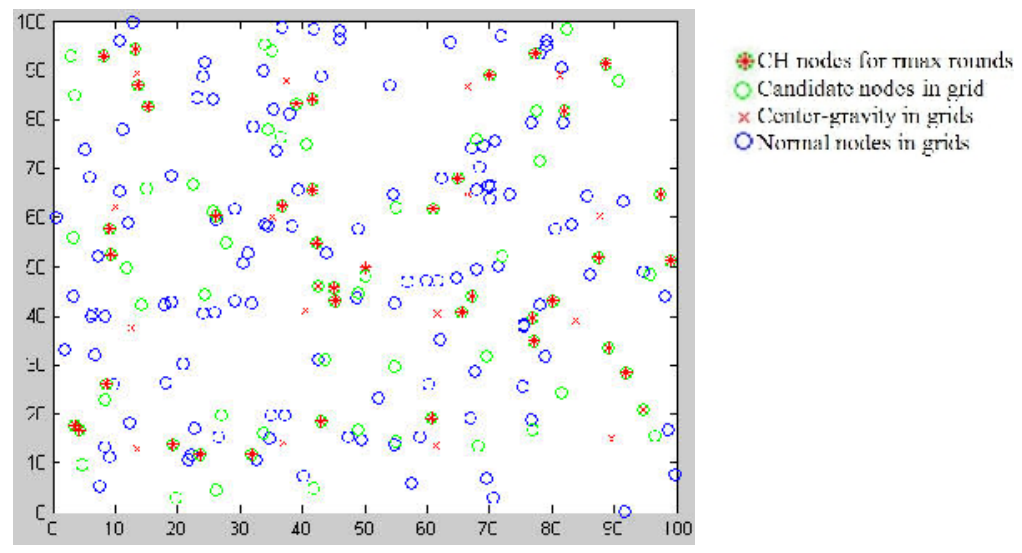

Figure 5. Gridding and find CHs in SLGC

\subsection{The Number of Alive Nodes}

Figure 6 shows the total number of alive nodes through simulation time. This figure shows that in SLGC the nodes have longer lifetime that in LEACH and SLGC decrease energy consumption in network. This result is as a selecting best $\mathrm{CHs}$ base on distance and thereshold of energy in grids for several rounds, then decrease energy consumption and volume of controlling message in network then increasing network lifetime.

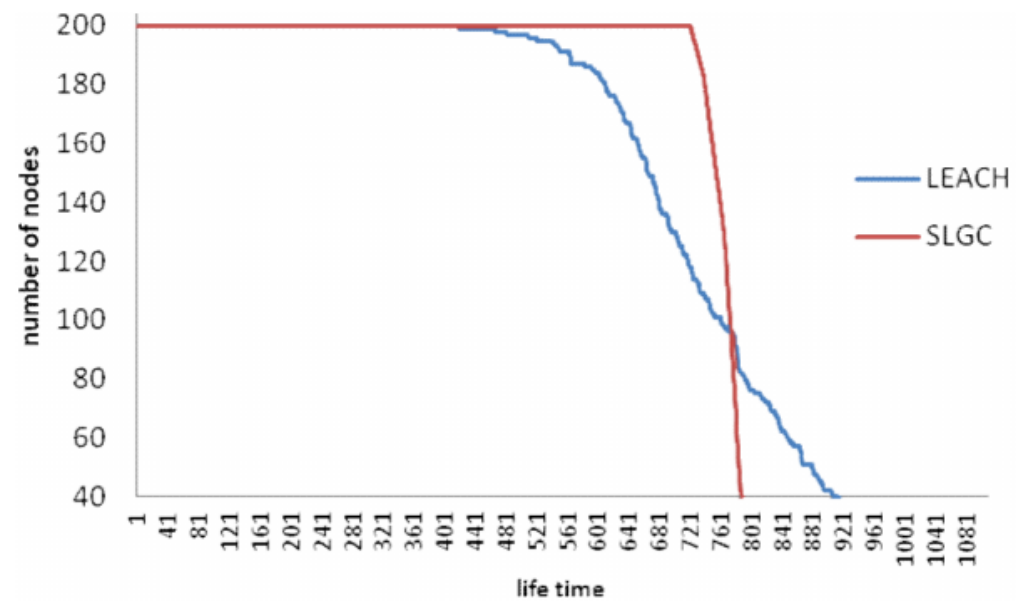

Figure 6. Total number of alive nodes in the SLGC and LEACH

\subsection{Network Lifetime with Different Number of Nodes}

We have compared SLGC in a network with a fixed size of $(100 \times 100)$ with a different number of nodes. In figure 7 shows the network lifetime until the first node dies and figure 8 shows lifetime until 30\% nodes die. This result is as a selecting best $\mathrm{CHs}$ base on distance and thereshold of energy in grids. Selecting best $\mathrm{CH}$ cause that increase network lifetime and first node dead later than LEACH. 


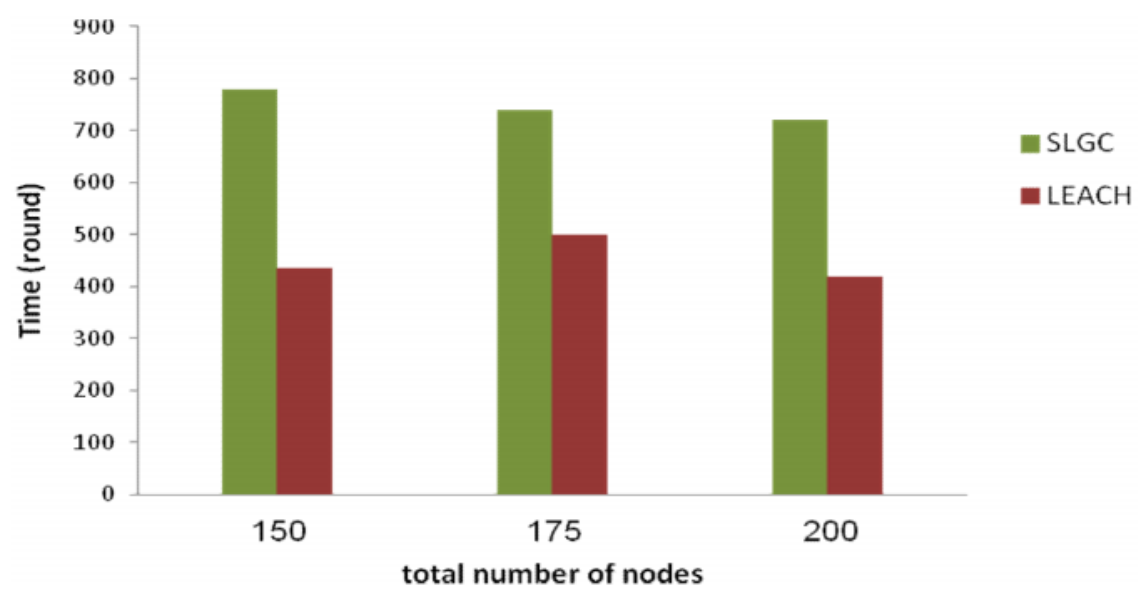

Figure 7. Network lifetime (first node die)

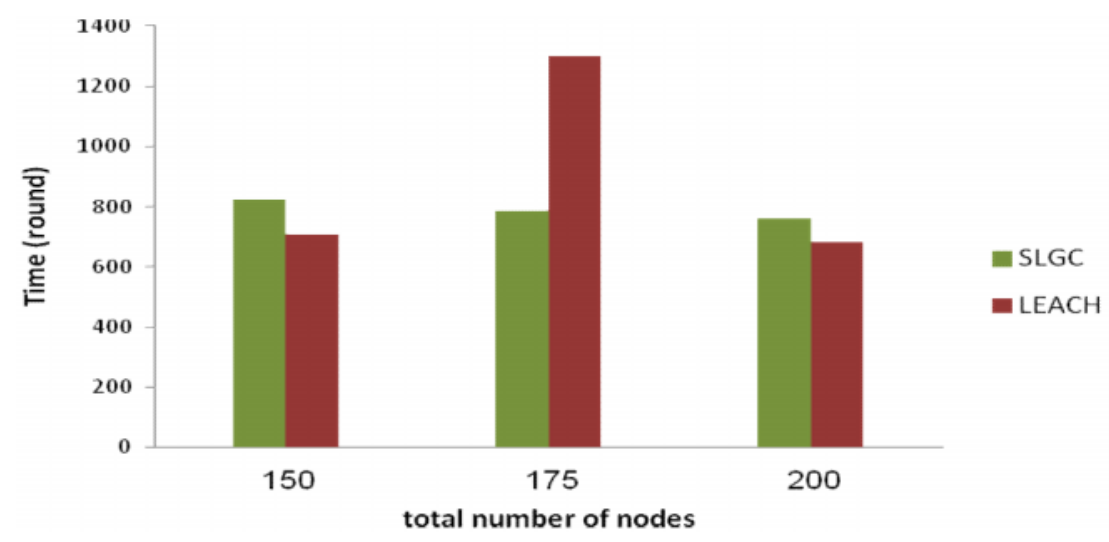

Figure 8. Network lifetime (30\% nodes die)

\section{Conclusions}

In Wireless Sensor Networks (WSNs) there are a large number of sensors that requiring careful architecture and management of the network. Decrease energy consumption and maximize Network lifetime are important parameters in designing WSNs. Clustering is one of the efficient methods in energy consumption by Cluster-Head in WSN. In this paper we have presented a new cluster routing algorithm to gridding wireless sensor network. We have shown that it is possible to create an efficient method for creating clusters in sensor network by selecting best Cluster Heads in each grid, $\mathrm{CHs}$ select base on maximum energy in grid and distance of node to center gravity of each grid, CHs aggregate the information from nodes and sending this data to BS, hence increasing network lifetime and decreasing energy consumption. Also SLGC algorithm with selecting $\mathrm{CHs}$ for next rounds reduces the volume of controlling message in network. We have improved the presented algorithm in an efficient and compared it with the LEACH method in cluster formation. Results show a higher efficiency level of network lifetime and decrease of energy consumption. 
International Journal of Computer Science, Engineering and Applications (IJCSEA) Vol.2, No.3, June 2012

\section{REFERENCES}

[1] Jeong, W., Nof, S.Y.: Performance evaluation of wireless sensor network protocols for Industrial applications. Journal of Intelligent Manufacturing 19(3), 335-345 (2008)

[2] D.chen and P.K.varshney," QOS support in Wireless Sensor Networks: A Survey". In proc of International Conference on Wireless Networks (ICWN '04), pp. 227-233, Las Vegas,Nev.. USA June 2004.

[3] K. Akkays and M. Younis, “A Survey on Routing Protocols for Wireless Sensor Network.” Elsevier Ad Hoc Network Journal, vol.3, no.3, May, 2005, pp. 325-349.

[4] Stanislava Soro, Wendi B. Heinzelman, "Cluster head election techniques for coverage preservation in wireless sensor networks", Ad Hoc Networks 7 (2009), pp. 955-972

[5] Karl H, Wiling A. Protocols and architectures for wireless sensor networks. Chichester: Wiley; 2005.

[6] Arboleda LMC, Nasser N. Comparison of clustering algorithms and protocols for wireless sensor networks. In: Proceedings of the Canadian conference on electrical and computer engineering; 2006. p. 1787-92.

[7] C.Konstantopoulos,A.Mpitziopoulos, D.Gavalas, G.Pantziou, "Effective Determination of Mobile Agent Itineraries for Data Aggregation on Sensor Networks", IEEE Transaction On Knowledge and Data Engineering, Vol. 22, NO. 12, December 2010

[8] S. Bandyopadhyay and E. J. Coyle, "Minimizing communication costs in hierarchically-clustered networks of wireless sensors," Computer Networks, vol. 44, no. 1, pp. 1-16, Jan. 2004.

[9] Y. Ma and J. H. Aylor, "System lifetime optimization for heterogeneous sensor networks with a hubspoke topology," IEEE Trans. Mobile Computing, vol. 3, no. 3, pp. 286-294, July-Sep. 2004.

[10] W.R.Heinzelman,A.Chandrakasan,H.Balakrishnan,"Energye fficient communication protocol for wireless sensor networks", In: Proceedings of the 33rd Hawaii International Conference on System Science, vol. 2, 2000.

[11] Abbasi, A.A., Younis, M.: A survey on clustering algorithms for wireless sensor networks. Computer Communications 30, 2826-2841 (2007)

[12] Arash Ghorbannia Delavar, javad Artin,Mohammad Mehdi Tajari, " RCSDN: a Distributed Balanced Routing Algorithm with Optimized Cluster Distribution"2011 3rd International Conference on Signal Acquisition and Processing (ICSAP 2011)

[13] Jiaxi you,Qi han, Dominik lieckfeldt, jakob Salzmann, Dirk timmermann, "Virtual position based geographic routing for wireless sensor networks ",Elsevier ,Computer communications 33(2010), 1255-1265

[14] Wang, Q., Yang, W." Energy consumption model for power management in wireless sensor networks". In: 4th Annual IEEE communications society conference on sensor, meshand ad hoc communications and network, SECON 2007 (2007)

[15] Ameer Ahmed Abbasi, Mohammad Younis , " A survey on clustering algorithms for wireless sensor networks" Elsevier, computer communications 30 (2007) 2826-2841

[16] Ali Chamam, Samuel Pierre. " A distributed energy-efficient clustering protocol for wireless sensor networks", Elsevier ,Computers and Electrical Engineering 36 (2010) 303-312

[17] N. Dimokas, D. Katsaros, Y.Manolopoulos, " Energy-efficient distributed clustering in wireless sensor networks", Elsevier, Journal of Parallel and Distributed Computer. 70 (2010) 371_383

[18] Alippi C, Anastasi G, Francesco MD, Roveri M. An adaptive sampling algorithm for effective energy management in wireless sensor networks with energy-hungry sensors. IEEE Trans Instrum Meas 2010;59(2):335-44.

[19] Sutar US, Bodhe SK. Energy efficient topology control algorithm for multi-hop ad-hoc wireless sensor network. In: Proc. 3rd IEEE international conference on computer science and information technology (ICCSIT), Chengdu, China; July 2010.

[20] Wang H, Agoulmine N, Ma M, Jin YL. Network lifetime optimization in wireless sensor networks. IEEE J Sel Areas Commun 2010;28(7):1127-37.

[21] Wei Kuang Lai, Chung Shuo Fan, Lin Yan Lin, " Arranging cluster sizes and transmission ranges for wireless sensor networks",Elsevier , Information Sciences 183 (2012) 117-131 
International Journal of Computer Science, Engineering and Applications (IJCSEA) Vol.2, No.3, June 2012

\section{Authors}

Arash Ghorbannia Delavar received the MSc and Ph.D. degrees in computer engineering from Sciences and Research University, Tehran, IRAN, in 2002 and 2007. He obtained the top student award in Ph.D. course. He is currently an assistant professor in the Department of Computer Science, Payam Noor University, Tehran, IRAN. He is also the Director of Virtual University and Multimedia Training Department of Payam Noor University in IRAN. Dr.Arash Ghorbannia Delavar is currently editor of many computer science journals in IRAN. His research interests are in the areas of computer networks, microprocessors, data mining, Information Technology, and E-Learning.

[Somayrh Shamsi received the BS degree in computer engineering from Bu-Ali Sina University, Hamedan, Iran, in 2007. Now she is a student the MS degree in the department of Computer Science in Payam Noor University, Tehran, IRAN. Her research interests include computer networks, wireless communication, mobility sensor network.

Nafise Mirkazemi received the BS, in 2007 and now, she is a Student the MS degree in the department of Computer Science in Payam Noor University, Tehran, IRAN. Her research interests include computer networks, wireless communication, mobility sensor network.

Javad Artin received the BS, in 2007 and now, he is a Student the MS degree in the department of Computer Science in Payam Noor University, Tehran, IRAN. His research interests include computer networks, wireless communication, Genetic algorithm, and Fuzzy logic 